\title{
Pacific Futures de Michaël Powles
}

\section{Raymond MAYER}

\section{(2) OpenEdition}

\section{Journals}

\section{Édition électronique}

URL : http://journals.openedition.org/jso/2012

DOI : 10.4000/jso.2012

ISSN : 1760-7256

\section{Éditeur}

Société des océanistes

\section{Édition imprimée}

Date de publication : 15 décembre 2008

Pagination : 337-338

ISBN : 978-2-85430-012-3

ISSN : 0300-953x

\section{Référence électronique}

Raymond MAYER, "Pacific Futures de Michaël Powles », Journal de la Société des Océanistes [En ligne], 126-127 | Année 2008, mis en ligne le 01 décembre 2008, consulté le 24 septembre 2020. URL : http:// journals.openedition.org/jso/2012 ; DOI : https://doi.org/10.4000/jso.2012

Ce document a été généré automatiquement le 24 septembre 2020

(c) Tous droits réservés 


\title{
Pacific Futures de Michaël Powles
}

\author{
Raymond MAYER
}

\section{RÉFÉRENCE}

Powles Michaël (ed.), 2006, Pacific Futures, The Australian National University, Pandanus Books, 274 p., bibliogr., tabl., index.

1 Ancien ambassadeur de Nouvelle-Zélande en Chine et à l'onu, président-fondateur de la Fondation néo-zélandaise pour la Coopération dans le Pacifique, Michaël Powles a pris l'initiative de publier les communications des scientifiques et des politiques faites au Forum des îles du Pacifique qui avait tenu son sommet de 2004 à Apia. Cette instance, comme on le sait, concerne essentiellement les quatorze États souverains du Pacifique insulaire et exclut ordinairement de son champ de compétence tous les territoires océaniens administrés par des pays tiers (USA, France, Royaume-Uni, Indonésie, Chili) à l'exception de ceux qui le sont par l'Australie ou la Nouvelle-Zélande. Néanmoins, les textes abordent des questions d'intérêt général en fournissant - de manière aléatoire et irrégulière, il est vrai - des données sur l'ensemble des populations et territoires de l'Océanie, débordant ainsi fréquemment du cadre restreint de leur juridiction spécifique.

2 Comme l'indique le titre de l'ouvrage, la visée prospective est l'idée directrice des communications. Celles-ci s'articulent autour de trois thèmes: les questions constitutionnelles et juridiques, les questions économiques, sociales et culturelles et, enfin, les questions de coopération régionale qui constituent le cœur de cible du Forum ou, si l'on préfère, ses attendus majeurs. L'évolution non seulement prévisible mais préconisable dans le cadre de chacun des domaines abordés donne à l'ouvrage sa cohérence fondamentale.

Dans la première partie de l'ouvrage, on aura ainsi plaisir à découvrir l'analyse (pp. 10-19) que John Henderson, professeur associé de science politique à l'université de Canterbury à Christchurch, consacre aux difficultés d'une «bonne gouvernance » dans des États érigés artificiellement (p. 13) et héritant des handicaps générés par la période 
coloniale - l'auteur cite en particulier le transfert des populations indiennes à Fidji modifiant fondamentalement le ratio démographique initial et créant d'impossibles équilibres politiques À l'exception des îles Gilbert et Ellice (les premières se rattachant à une entité micronésienne, les secondes constituant une entité polynésienne), peu d'archipels purent de même modifier les frontières héritées de la colonisation. Sur la base de l'existant, Henderson analyse les avantages comparés entre régimes parlementaires et régimes présidentiels et conclut qu'en dépit des coups d'État et émeutes qui peuvent donner l'impression d'une "africanisation" de certains États océaniens, la plupart des quatorze États indépendants du Pacifique ont réussi à établir d'authentiques démocraties.

4 Revenant sur la question des compromis possibles entre régimes traditionnels et nouvelles aspirations démocratiques (pp. 20-35), Teresia Teaiwa, directrice de programme à l'université Victoria de Wellington, et Malakai Koloamatangi, en poste à l'université de Canterbury à Christchurch, examinent l'évolution des rapports de force à Tonga et à Kiribati, observant les mauvaises dérives du pouvoir monarchique dans le premier cas et, au contraire, l'accession à une relative stabilité par le refus de toute solution de type autocratique dans le second cas (p. 30). Les situations sont donc plus variées dans le Pacifique qu'on ne le croit et ne sauraient être ramenées à un modèle unique. Le tableau sur les systèmes politiques en exercice dans le Pacifique (pp. 32-35) est tout à fait éclairant sur la diversité des situations que l'on peut y rencontrer.

L'étude du cas samoan (pp.36-49) proposée par Lau Asofou So'o, professeur à l'université de Samoa, est exemplaire d'une critique et autocritique rigoureuses appliquées à l'intégration d'un système traditionnel de chefferie à titres (matai) fondé sur cinq niveaux de pouvoir (familles étendues, 238 villages, 11 sub-districts, districts et un gouvernement national pour 175000 habitants) à un modèle de démocratie parlementaire. Chaque niveau de pouvoir a de fait été respecté dans la pyramide politique des quarante premières années d'indépendance (1962). Néanmoins l'équilibre entre pouvoir électif et pouvoir hérité par titre reste une question ouverte.

6 L'application des droits de l'Homme dans la culture fidjienne pose un problème de compatibilité de systèmes qui ne sont pas inconciliables, selon Ratu Joni Madraiwiwi, juriste et vice-président de Fidji en 2005 (pp. 50-52). L'auteur considère en effet que la société fidjienne traditionnelle opère largement par consensus (p.50). Quand bien même le pouvoir appartient aux chefs (turaga), un vieil adage fidjien dispose : «le peuple est le peuple des chefs, les chefs sont les chefs du peuple», établissant un équilibre de principe entre le statut des uns et des autres. Dans la pratique, l'auteur plaide pour un dialogue constructif visant à rendre exécutoires les droits de l'homme en fonction du contexte local.

7 Dans la deuxième partie de l'ouvrage, la perte des langues et cultures océaniennes retient l'attention de Galumalemana A. Hunkin et Fepuleai Lasei J. F. Mayer, tous deux ayant notablement travaillé à l'intégration de la langue samoane dans les programmes universitaires (pp.62-71). Ils rappellent les chiffres qui donnent l'ampleur de la question linguistique et culturelle dans le Pacifique: 832 langues en Papouasie Nouvelle-Guinée, 226 en Mélanésie, 20 en Micronésie et 38 en Polynésie (p.62). Ils analysent le processus de la déperdition des langues et s'étendent, dans une visée prospective, sur la restauration durable des langues maorie et hawaiienne comme langues maternelles préscolaires en Nouvelle-Zélande et à Hawaii (pp. 68-69). 
Deux chapitres sont dévolus à l'efficience des traités internationaux et régionaux dans le Pacifique et à la discussion de l'opportunité du modèle néo-libéral dans le contexte océanien. Un chapitre (pp. 111-126) est consacré par T.K. Jayaraman, professeur associé à l'université du Sud Pacifique de Suva, à l'hypothèse d'une monnaie unique pour l'ensemble des États du Pacifique. L'auteur conclut sur la faiblesse des échanges commerciaux entre les îles et sur leur dépendance par rapport aux économies australienne et néo-zélandaise. L'émigration de la main-d'œuvre insulaire vers l'Australie et la Nouvelle-Zélande fait l'objet de l'étude de Satish Chand (pp. 127-141), qui enseigne les sciences économiques à l'université de Canberra. Il dresse des tableaux de flux migratoires, des taux de non-retour, des revenus per capita et des contributions des émigrés au développement de leurs îles d'origine. Il interroge la faiblesse des performances économiques des jeunes États océaniens. Vijay Naidu, professeur à l'École des sciences de la Terre à l'université de Wellington, poursuit une enquête similaire sur les défis de l'aide au développement dans le contexte insulaire (pp. 142-163). Analysant les modalités de l'aide bilatérale et multilatérale, il remet en cause les paradigmes classiques et préconise un " développement participatoire » fondé sur des partenariats renforcés (p. 156).

Le changement climatique et ses dramatiques conséquences sur certaines îles basses sont traités par Graham Sem, en charge du secrétariat du programme régional des Nations unies sur le changement climatique. Son article (pp. 164-181) évoque plusieurs scénarios et appelle des solutions adaptées à des besoins impérieux liés en particulier au relèvement du niveau de la mer et à la vulnérabilité des économies insulaires dans les nouveaux contextes climatiques prévisibles.

La troisième partie de l'ouvrage est tout entière tournée vers des esquisses d'avenir pour la région Pacifique. Sont abordées les questions de coopération régionale (Richard Herr, pp. 184-194), fondée ou non sur les substrats culturels (Ron Crocombe, pp. 195-203), sur des perspectives communautaires (Greg Fry, pp. 204-215), sur l'exercice renforcé ou non de la souveraineté insulaire (Transform Aqorau, pp. 216-223). Une quatrième partie regroupe les vœux que quelques leaders politiques placent dans l'avenir de la région Pacifique (pp. 225-239). On doit dire que l'ensemble de l'ouvrage, même s'il présente çà et là des hétérogénéités dans le système documentaire inégalement appliqué à toutes les entités politiques de l'Océanie, répond à des préoccupations prospectives dont nul ne saurait contester l'urgence et la nécessité scientifiques.

\section{AUTEURS}

\section{RAYMOND MAYER}

université Lyon II 